\title{
SMILE-BR: aplicação de conceitos de gamificação em um ambiente de aprendizagem baseado em questionamento
}

\author{
Rômulo Martins França, Eliseo Berni Reategui \\ Universidade Federal do Rio Grande do Sul - Av. Paulo Gama, 110 - prédio 12105 - $3^{\circ}$ \\ andar, Porto Alegre, RS, Brasil \\ romulomf@gmail.com, eliseoreategui@gmail.com
}

\begin{abstract}
One of the limitations of computational learning environments is related to the engagement of students, a fundamental factor for learning processes. A new perspective that tackles this problem and incorporates games' elements to software in different areas is Gamification. This paper presents a study about the use of Gamification concepts in an Inquiry-Based Learning Environment. While the inquiry-based learning approach focuses on the development of investigation habilities, the Gamification elements introduce principles related to collaborative and competition-based learning.
\end{abstract}

Resumo: Uma das limitações que se observa em ambientes computacionais voltados à Educação diz respeito ao engajamento dos alunos, um fator fundamental aos processos de aprendizagem. Uma nova perspectiva que trata essa questão e incorpora mecânicas de jogos a softwares de diversas áreas é a Gamificação. Este artigo apresenta um estudo sobre o uso dos conceitos de Gamificação em um Ambiente de Aprendizagem baseado em Questionamento. Enquanto a abordagem de aprendizagem baseada em questionamento foca no desenvolvimento de habilidades investigativas nos alunos, os elementos de Gamificação introduzem princípios relacionados à aprendizagem baseada em colaboração e competição.

\section{Introdução}

Cada vez mais, um maior número instituições educacionais emprega softwares educativos como facilitadores do processo de ensino e aprendizagem (Braga, 2006). Diversas abordagens de aprendizagem são utilizadas como alternativa a métodos mais tradicionais. Um destes exemplos é a abordagem de Aprendizagem baseada em Questionamento (do inglês Inquiry-based Learning), definida como uma abordagem de aprendizagem guiada pelo processo de elaboração de perguntas pelos próprios aprendizes (Barret et al., 2005). Nesta abordagem, busca-se instigar os aprendizes à elaboração de questões sobre determinado tema, como forma de levá-los a desenvolver suas próprias investigações sobre o assunto.

Apesar disso, uma das limitações que se observa em ambientes computacionais voltados à Educação está relacionada ao engajamento dos alunos, um fator fundamental no que diz respeito aos processos de aprendizagem (Killi, 2005). Uma nova perspectiva que trata essa questão e incorpora mecânicas de jogos a softwares de diversas áreas vem ganhando força, sendo conhecida como Gamificação, do inglês Gamification. Aldrich (2009) define o conceito como a utilização de elementos dos jogos no desenvolvimento de outros artefatos, buscando maior motivação e engajamento dos usuários. 
Já Lee (2011) complementa e aponta o elevado potencial para aplicação da Gamificação na área da educação. O autor enfatiza que o objetivo desta abordagem não é "ensinar com os jogos", mas sim usar elementos de jogos como forma de promover a motivação e o envolvimento dos alunos.

A Gamificação compartilha elementos do design dos jogos para lançar desafios, usar estratégias, obter pontos para atingir determinados objetivos, liberar acesso a itens bloqueados, conquistar espaço, ganhar visibilidade e recompensas, medalhas e prêmios (BUNCHBALL, 2012). Os ambientes Busuu ${ }^{1}$ e Livemocha ${ }^{2}$ são exemplos de ambientes voltados ao ensino e aprendizado de idiomas que possuem elementos de Gamificação.

Este artigo apresenta um estudo sobre o uso dos conceitos de Gamificação em um Ambiente de Aprendizagem baseado em Questionamento (SMILE), desenvolvido originalmente pela Universidade de Stanford. Será apresentada uma nova proposta de ambiente (SMILE-BR), sendo descritas as principais funcionalidades e mecanismos dos jogos empregados como forma de envolver os estudantes em dinâmicas que fomentam a interação e colaboração.

Com o propósito de contribuir no projeto SMILE, Acosta e Reategui (2012) propuseram a integração de funcionalidades de recomendação de conteúdo ao ambiente. Este trabalho dá sequência a tais pesquisas, propondo novas contribuições diante de aprendizes criados em mundo cada vez mais digital. Coloca-se, assim, o problema que motiva esta pesquisa: como os elementos dos jogos podem contribuir com $o$ engajamento dos aprendizes no desenvolvimento de atividades em um ambiente de aprendizagem baseado em questionamento?

As próximas seções estão divididas do seguinte modo: a seção 2 apresenta a aprendizagem baseada em questionamento como abordagem pedagógica alternativa a abordagens mais convencionais centradas no professor. A seção 3 apresenta o conceito de Gamificação, colocando-o como um elemento capaz de envolver a nova geração de aprendizes tão familiarizada com o universo dos games. A seção 4 descreve brevemente o ambiente SMILE, desenvolvido pela Universidade de Stanford, e apresenta o ambiente proposto neste artigo com uso da Gamificação, intitulado de SMILE-BR. A seção 5 é a discussão sobre a aplicação dos conceitos de Gamificação no ambiente SMILE-BR. Por fim, a seção 6 apresenta as contribuições, considerações finais e possibilidades para trabalhos futuros.

\section{Aprendizagem Baseada em Questionamento}

Nos Estados Unidos, o conceito da Aprendizagem baseada em Questionamento tem uma longa história na rede pública de ensino, especialmente no ensino de ciências (Justice, 2007). Ele é referido por diversos teóricos na Educação como um conceito fundamentado na importância dos aprendizes elaborarem seus próprios questionamentos com relação aos temas sendo tratados. Na pesquisa de Dewey (1896), a Aprendizagem baseada em Questionamento reflete na crença de que os estudantes necessitam desenvolver o pensamento crítico ao invés de habilidades de memorização.

A Aprendizagem Baseada em Questionamento pode ser definida como um conjunto de práticas de sala de aula que promovem a aprendizagem dos alunos através da

\footnotetext{
${ }^{1}$ http://www.busuu.com

${ }^{2}$ http://www.livemocha.com
} 
investigação orientada e que, cada vez mais independente, abordam questões e problemas complexos (Lee, 2004). Trata-se de uma abordagem que promove o pensamento crítico, o desenvolvimento da habilidade de investigação e das responsabilidades pela própria aprendizagem.

A Aprendizagem baseada em Questionamento refere-se a práticas pedagógicas planejadas pelos professores para promover o desenvolvimento de habilidades de ordem intelectual e acadêmica, através de questões elaboradas pelos próprios estudantes (Hudspith e Jenkins 2001; Justice et al., 2007).

Spronken-Smith (2007) se refere à abordagem da Aprendizagem baseada em Questionamento como uma pedagogia que permite aos alunos vivenciarem melhor os processos de criação de conhecimento. Ela está sob a esfera das abordagens indutivas de ensino e aprendizagem, assim como uma gama de outros métodos de ensino como a Aprendizagem baseada em Problema, Aprendizagem baseada em Projeto e a Aprendizagem pela Descoberta (Prince e Felder, 2006).

As abordagens indutivas de ensino e aprendizagem iniciam com um conjunto de observações ou dados para interpretação, ou um problema complexo do mundo real. Dado este ponto de partida, os alunos estudam os problemas que geram uma necessidade de coleta de fatos, procedimentos e diretrizes. Spronken-Smith (2007) apresenta algumas características comuns aos métodos indutivos, tais como: a) a aprendizagem é centrada no aluno e não na apresentação de conteúdos ou conhecimentos; b) a aprendizagem ativa é vista sob a ótica do "aprender fazendo" e que pode envolver, por exemplo, alunos discutindo questões e resoluções de problemas; c) as habilidades de autoaprendizagem são priorizadas, na qual os alunos carregam mais responsabilidades pela sua própria aprendizagem. d) uma base teórica construtivista que norteia a construção dos significados da realidade pelos próprios alunos. e) muitos desses métodos indutivos também se fundamentam na cooperação/colaboração.

A abordagem de Aprendizagem baseada em questionamento tem sido empregada em diferentes Ambientes e Comunidades Virtuais de Aprendizagem, como, por exemplo, o ambiente SMILE (Stanford Mobile Inquiry-based Learning Environment), desenvolvido na Universidade de Stanford. O ambiente tem o objetivo de facilitar o desenvolvimento de atividades seguindo essa abordagem por meio do uso de dispositivos móveis. Entende-se que as perguntas criadas pelos próprios alunos, seguindo preceitos da aprendizagem baseada em questionamento, possam desempenhar um papel importante no processo de aprendizagem na medida em que os levam ao questionamento, à reflexão e à resolução dos próprios questionamentos (Acosta e Reategui, 2012).

Muitos outros trabalhos na área têm-se apoiado na abordagem de Aprendizagem baseada em Questionamento. Yoder (2003), que apresenta casos de construção de webquests fundamentadas neste tipo de aprendizagem. Mott e Lester (2006) descrevem um ambiente de aprendizagem centrado no desenvolvimento de narrativas e ancorado na aprendizagem baseada em questionamento. Já Lim (2004) discute questões e problemas relacionados ao emprego da abordagem na web, um ambiente considerado amplo, pouco estruturado e complexo.

Apesar dos resultados significativos destes projetos, no que diz respeito à capacidade de tal abordagem em desenvolver nos alunos o pensamento crítico e a capacidade investigativa, sabe-se hoje em dia do desafio da escola em envolver os estudantes em atividades de aprendizagem motivadoras. Neste contexto, o projeto aqui apresentado 
propõe a introdução da gamificação em um ambiente de aprendizagem baseado em questionamento como forma de engajar os alunos no desenvolvimento de suas próprias investigações em um ambiente colaborativo.

\section{Gamificação}

A Gamificação diz respeito ao uso de técnicas de jogo para tornar atividades mais envolventes e divertidas (Kim, 2010). Egenfeldt-Nielsen (2011) afirma que a Gamificação busca integrar as dinâmicas de jogos dentro de um serviço, comunidade ou campanha, a fim de incentivar um determinado comportamento, atitude ou habilidade dos indivíduos. Deterding et al. (2011) reforçam esta posição dizendo que a inserção de elementos de jogos a contextos não lúdicos tem o potencial de gerar, entre os participantes, um nível de envolvimento semelhante ao que é conseguido com os jogos

Kapp (2007) define Gamificação de forma semelhante, mas enfatiza o uso da mecânica, estética e raciocínio correntes nos jogos, como forma de engajar as pessoas, conduzi-las na solução de problemas e promover os processos de aprendizagem.

De acordo com Liu, Alexandrova e Nakajima (2011), para usar a Gamificação em um ambiente não relacionado aos jogos, a ideia é criar-se um ciclo de Gamificação. A interação inicia a partir de um objetivo ou desafio claro com uma condição de vitória específica. Toda vez que o usuário alcançar uma determinada meta, algumas recompensas são dadas nesse sentido, o que é normalmente suportado por um sistema de pontos (pontuação, moeda virtual, pontos de experiência etc). Com base no sistema de pontos e históricos de conquistas, um quadro de líderes (globais ou parciais) e emblemas é fornecido aos jogadores para motivar a colaboração e a competição.

A maior parte das aplicações que empregam conceitos de Gamificação estabelece alguma forma de recompensa pelas atividades realizadas pelo usuário. Estes sistemas utilizam as mecânicas de jogos mais comuns, tais como emblemas, níveis, quadros de liderança, metas e pontos. Essa estrutura também é definida por Nicholson (2012) como $B L A P$ da Gamificação (Badges, Leaderboards, Achievements e Points) que correspondem a Emblemas, Quadro de Líderes, Conquistas e Pontos.

$\mathrm{Na}$ proposta apresentada neste artigo, buscou-se empregar alguns destes elementos no ambiente de aprendizagem baseado em questionamento apresentado na próxima seção.

\section{Ambiente SMILE-BR}

O projeto SMILE da Universidade de Stanford, que surgiu em 2009 e já foi empregado em mais de 20 países, está relacionado à aprendizagem baseada em questionamento mediada pela tecnologia, cujo objetivo principal é possibilitar aos alunos a criação e compartilhamento de questões relacionadas a um determinado assunto (KIM et al., 2011). Ela é uma ferramenta para gerenciamento das perguntas e respostas elaboradas pelos alunos, com foco no emprego de dispositivos móveis. Resultados em diversos experimentos com o uso da ferramenta demonstraram o envolvimento e bom desempenho dos alunos nas atividades propostas.

Já a proposta do SMILE-BR é um complemento do ambiente SMILE original, superando limitações de motivação e engajamento dos alunos por meio de elementos de jogos e também por armazenar dados na web para possibilitar o acesso dos professores e estudantes aos projetos, a partir de qualquer local com acesso à internet. 
O SMILE-BR também é baseado na Abordagem baseada em Questionamento com lançamentos de desafios entre os alunos, a partir das temáticas levantadas pelos professores, contribuindo com a aplicação dos conceitos de Gamificação. Ele é composto por dois módulos: professor e aluno.

\subsection{Módulo do Professor}

O módulo do professor é composto pelo gerenciamento de temáticas e visualização dos desafios dos alunos. A área da temática é o local em que o professor monta uma página multimídia com conteúdo sobre um determinado assunto, instigando os alunos a elaborarem questionamentos sobre a temática abordada na forma de desafios.

a) Cadastro de temática: à esquerda na interface da figura 1 é utilizada pelo professor para criar uma temática com título, conteúdo e hiperlinks para adicionar anexos (imagens, documentos, vídeos, áudios). O material criado também pode ser compartilhado via Facebook. Na definição da temática o professor escolhe as regras para determinar a pontuação dos alunos e alimentar dados relacionados à Gamificação. O professor determina as regras para avaliação dos questionamentos (desafios) lançados pelos alunos, que pode ser de quatro formas: 1) avaliação por outros alunos: através do recurso de estrelas com nível de um a cinco totalizando até 5 pontos em cada avaliação; 2) número de curtidas no Facebook: o aluno recebe um ponto por "curtida" de outros colegas; 3) avaliação do professor: também através do recurso de estrelas com nível de um a cinco, só que o aluno recebe dois pontos por estrela marcada, totalizando até 10 pontos (5 estrelas); 4) avaliação híbrida (aluno e professor): seguem as regras estabelecidas conforme já apresentado nos itens anteriores.

b) Listar temáticas: ao centro da figura 1, o professor pode visualizar temáticas criadas por ele e por outros professores com o título, data de criação e número de alunos que lançaram desafios. Além disso, o professor tem a opção de "Criar" uma temática.

c) Visualizar temática: à direita da figura 1 são listadas as contribuições dos alunos em uma temática com o nome do aluno, número de participantes e "curtidas" no Facebook.
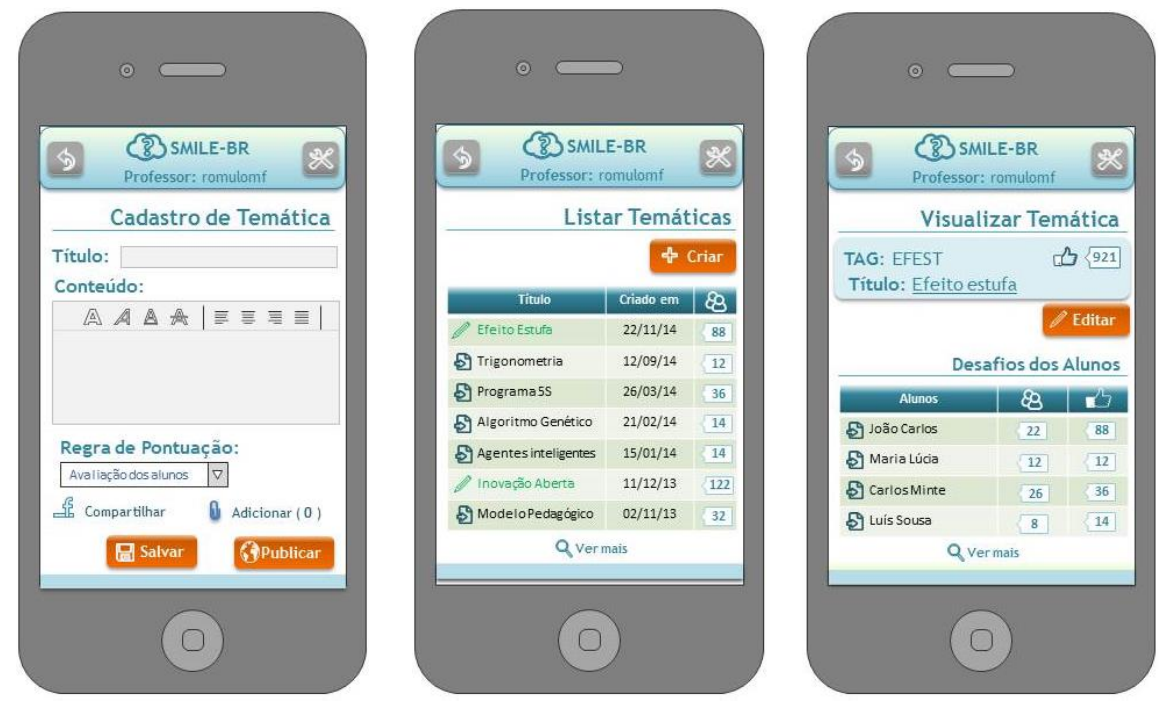

Figura 1 - a) Interface para cadastrar as temáticas (esquerda); b) listar as temáticas criadas (centro); c) visualizar uma temática específica (direita) 


\subsection{Módulo do Aluno}

Neste módulo os alunos criam os questionamentos como desafios e compartilham com os demais colegas, sendo criadas alternativas com respostas objetivas, seguindo a linha do projeto SMILE original. As seguintes funcionalidades compõem este módulo:

a) Listar temáticas: à esquerda na interface da figura 2 o aluno pode visualizar as temáticas criadas pelos professores. As informações que constam nesta tela apresentam o título da temática, data de criação e número de alunos participantes.

b) Visualizar temática: ao centro na interface da figura 2 são listados os desafios dos outros alunos em uma temática, apresentados em uma tabela composta por um ícone que dá acesso ao desafio, apresentando nome do aluno, número de participantes e curtidas no Facebook. Além disso, tem a opção de "Lançar Desafio" na temática.

c) Lançar desafio: à direita na interface da figura 2, o aluno visualiza o código de identificação da temática (TAG), título e o número de curtidas da temática. O aluno insere uma descrição com o seu questionamento e as alternativas (opções) de resposta. Ele deve especificar qual é a alternativa correta. Além disso, os hiperlinks, anexos (documentos, vídeos, áudios), imagens, citação a outros alunos que já contribuíram e compartilhamento via Facebook podem ser adicionados ou realizados.
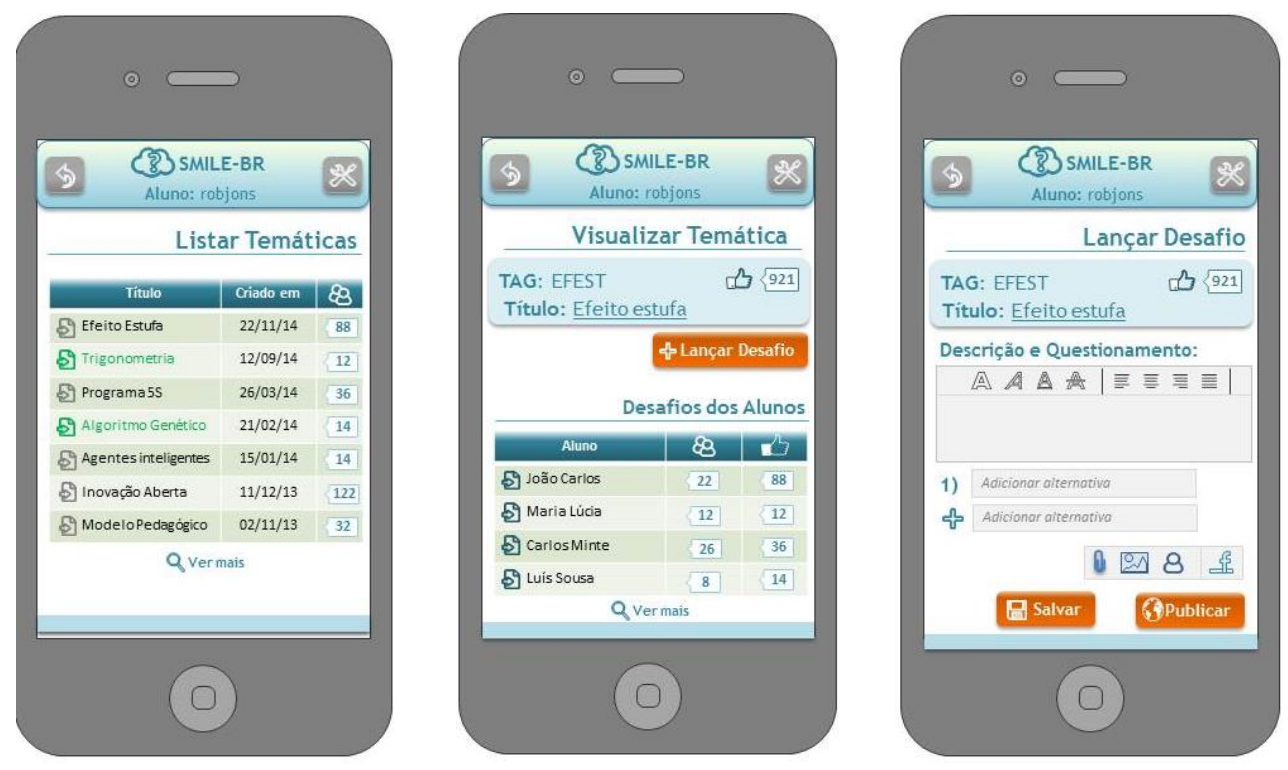

Figura 2 - a) Interface para listar as temáticas criadas (esquerda); b) visualizar uma temática específica (centro); c) lançar um desafio (direita)

d) Participar do desafio: à esquerda na interface da figura 3 abaixo, o aluno acessa o desafio lançado por outro colega, sobre a temática abordada pelo professor, e responde o questionamento escolhendo uma das alternativas disponíveis. Além de pontuar recebendo avaliação do seu desafio estabelecido pelo professor, o aluno marca 10 pontos por desafios solucionados de forma correta. Nesta tela, o aluno pode ainda visualizar anexos, imagens e citações feitas a outros colegas pelo criador do desafio. No final da tela, ele ainda tem a opção de avaliar o desafio (questionamento) lançado através das estrelas.

e) Ver Perfil, Ranking, Recompensas e Objetos de Aprendizagem: ao centro na interface da figura 3 abaixo, pode ser visualizado o perfil do aluno. Conforme eles vão 
ganhando pontos nos desafios e nas avaliações o ambiente vai desbloqueando novos conteúdos, recursos e funcionalidades. Quanto mais experiência (pontos) os alunos tiverem, mais funcionalidades e conteúdos terão em seu perfil. $\mathrm{O}$ ranking pode ser visualizado à direita da figura 03 , no qual a sua ideia não é colocar em evidência o aluno que mostrou mais conhecimento em determinada área, mais sim, valorizá-los pela sua participação, contribuição e engajamento no processo de construção de conhecimento. Em outras interfaces ainda podem ser visualizadas as recompensas desbloqueadas de acordo com a pontuação dos alunos e também links de objetos de aprendizagens (artigos, vídeos, livros) recomendados pelos professores nas temáticas para os alunos.
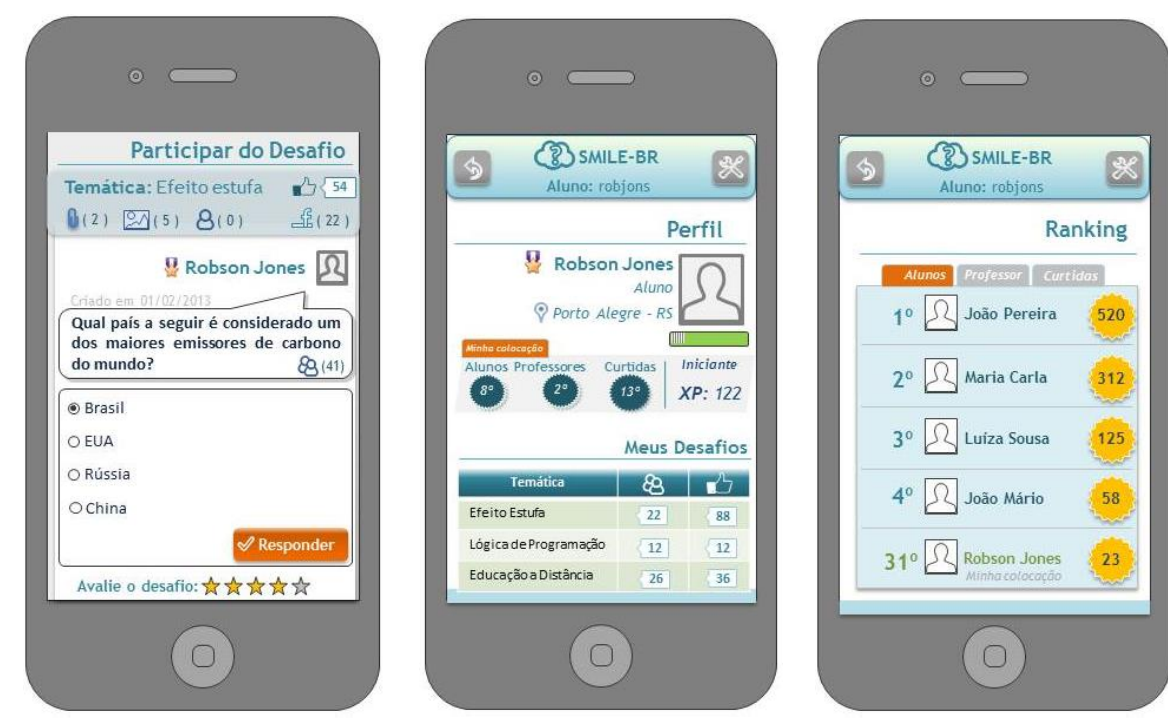

Figura 3 - a) Interface de participação do desafio (esquerda); b) perfil do aluno com experiência, colocação, emblemas e nível (centro); c) ranking de pontuação (direita)

\section{Discussão}

Esta seção discute o emprego dos conceitos-chaves da Gamificação no ambiente de aprendizagem proposto. Partimos da premissa de que os elementos da Gamificação introduzidos no ambiente podem promover a colaboração e competição entre os estudantes. Em ambos os casos, os elementos dos jogos acabam por atuar como fator motivacional que instiga os alunos a se empenharem na realização das atividades.

No que diz respeito à colaboração, inúmeros autores apontam sua importância nos processos de aprendizagem. Vygostky (1978), por exemplo, destaca que o desenvolvimento intelectual é alcançado quando os aprendizes são envolvidos em atividades de aprendizagem nas quais interagem uns com os outros.

Já Gohkale (1995) aponta que a aprendizagem colaborativa promove o desenvolvimento do pensamento crítico por meio da discussão, da explicação de ideias e da avaliação das ideias dos outros. Assim, o envolvimento dos aprendizes em interações significativas (ex. tomada de decisão, resolução de problema), mostra-se como um componente fundamental dos processos de aprendizagem.

No que diz respeito à competição, apesar de alguns educadores serem contrários à adoção de práticas competitivas, pesquisas mostram como estas pode apoiar os processos de aprendizagem. Burguillo (2010) define a aprendizagem baseada em competição como uma metodologia na qual a aprendizagem é alcançada através da 
competição, mas o resultado da aprendizagem é independente da pontuação do aluno na competição. Na pesquisa do autor este demonstrou que a utilização de competições amistosas promoveu melhorias no desempenho de um grupo de estudantes em um curso de programação, contemplando também estudantes com diferentes estilos de aprendizagem. Ediger (1996) destaca que a competição pode ser saudável ou destrutiva. Do mesmo modo, a colaboração pode ser positiva ou negativa. Tudo depende do contexto e da forma de condução das atividades em que os alunos são levados a competir ou colaborar.

No caso da proposta aqui apresentada, os elementos de Gamificação introduzidos acabam envolvendo os alunos tanto em situações de colaboração quanto de competição: os alunos podem colaborar na criação/resposta de questionamentos (desafios), mas também podem competir na obtenção de um melhor posicionamento quanto aos BLAPs (Emblemas, Quadro de Líderes, Conquistas e Pontos).

Mas é importante ressaltar que o conceito de Gamificação do ambiente aqui proposto abrange mais do que os elementos BLAP. Nicholson (2012) introduz a definição de "Gamificação com significado" (do inglês, meaningful gamification). De acordo com este conceito, em uma atividade não lúdica é importante criar um nível de envolvimento semelhante ao que se pode obter com os jogos, gerando uma experiência com significado que não depende apenas de recompensas extrínsecas.

No sentido de promover a motivação dos estudantes e envolvê-los na dinâmica proposta pelas recompensas no ambiente SMILE-BR, adotamos a visão de Zichermann (2011) que propôs uma estrutura que lida com a forma como os jogadores valorizam essas recompensas, conhecidas pelo acrônimo SASP: Status, Acesso, Poder e Coisas (do inglês, Status, Access, Power, Stuff). O Status simboliza as recompensas que alavancam os usuários dentro de um ranking interno do ambiente e permitem que outros estudantes vejam o seu progresso através de níveis e emblemas ou moedas virtuais. O Acesso significa a entrada privilegiada a partes internas ou externas do ambiente, às quais outros estudantes não teriam acesso. O Poder é a possibilidade de ter algum controle sobre o ambiente ou outros jogadores, por exemplo, sugerir novas funcionalidades para o ambiente, ou ser moderador em um fórum de discussão. Por fim, as Coisas são geralmente objetos cujo valor real é conhecido, nesta proposta adaptados para objetos de aprendizagem digitais.

\section{Considerações Finais}

As principais contribuições deste trabalho foram: a) a proposta $\mathrm{e}$ desenvolvimento do ambiente SMILE-BR combinando os conceitos educacionais da Aprendizagem baseada em Questionamento com os da Gamificação em um ambiente alinhado a teorias construtivistas. Nestas, o aluno é visto como sujeito ativo dos processos de aprendizagem; b) ampliação do projeto SMILE através de um ambiente com recursos que visam motivar e engajar os alunos nas atividades propostas.

Busca-se com o SMILE-BR expandir as possibilidades do ambiente de aprendizagem baseada em questionamento do ambiente SMILE original, no qual os alunos elaboram e compartilham questões sobre um determinado tema com outros colegas. Os questionamentos no ambiente foram contextualizados na forma de desafios e quesitos importantes da Gamificação, como Status, Acesso, Poder e Coisas, foram incorporados 
a partir da definição de pontuações, ranking, recompensas, níveis e desbloqueios de novas funcionalidades.

Como trabalhos futuros podemos citar o desenvolvimento de novas funcionalidades para o ambiente com recursos compartilhados nas redes sociais, bem como sua validação com turmas do Ensino Fundamental e Médio em um teste prático.

\section{Referências}

Acosta, Otávio C.; Reategui, Eliseo. (2012) Recomendação de Conteúdo em um Ambiente de Aprendizagem Baseado em Questionamento. RENOTE, v. 10.

Aldrich, C. (2009) Learning Online with Games, Simulations, and Virtual Worlds: Strategies for Online Instruction, Jossey-Bass, San Francisco.

Barrett, G., Selman, D. \& Thomas, G. (2005) Interprofessional Working in Health and Social Care: Professional Perspectives. Basingstoke: Palgrave Macmillan.

Braga, M. M. (2006) Design de software educacional baseado na teoria dos campos conceituais. Dissertação UFPE.

Bunchball. (2013) An Introduction to the Use of Game Dynamics to Influence Behavior. Acesso em 14 jan 2013. Disponível em: < http://www.bunchball.com/sites/default/files/downloads/gamification101.pdf>.

Burguillo, C. J. (2010) Using game theory and Competition-based Learning to stimulate student motivation and performance. Computers \& Education, 55(2), pp. 566-575.

Deterding, S., Sicart, M., Nacke, L., O'Hara, K., and Dixon, D. (2011) Gamification. using game-design elements in non-gaming contexts. In Proceedings of $\mathrm{CHI}$ Extended Abstracts, 2425-2428.

Dewey, J. (1896) The Reflex Arc Concept in Psychology. Psychological Review 3, pp 357-370.

Ediger, M. (1996) Cooperative learning versus competition: which one is better? Journal of Instructional Psychology, 23, pp. 204-300.

Egenfeldt-Nielsen, Simon. (2011) What Makes a Good Learning Game? Going beyond edutainment. eLearn Magazine.

Gokhale, A. A. (1995) Collaborative Learning Enhances Critical Thinking. Journal of Technology Education, 7(1).

Hudspith, B. and Jenkins, H. (2001) Teaching the art of inquiry. Ontario, Canada: Society for Teaching \& Learning in Higher Education. Green guide: No.3

Justice, C., Rice, J., Warry, W., Inglis, S., Miller, S., and S. Sammon. (2007) Inquiry in higher education: Reflections and directions on course design and teaching methods. Innovative Higher Education 31(4): 201-214.

Kapp, K. M. (2007) Gadgets, games, and gizmos for learning: tools and techniques for transferring know-how from boomers to gamers. [S.1.]: John Wiley and Sons.

Kiili, K. (2005) On educational game design: building blocks of flow experience. Tese de Doutorado. Tampere University. 
Kim, P., Goyal, A., Seol, S., Dodson, B., Lam, M. (2011) PocketSchool Interactive Learning Ad-hoc Network. To appear in the proceedings of IEEE International Conference on e-Education, Entertainment and e-Management.

Kohn, A. (1999) Punished by Rewards: The Trouble with Gold Stars, Incentive Plans, A's, Praise, and Other Bribes. Boston: Houghton Mifflin.

Lee, J. J. \& Hammer, J. (2011) Gamification in Education: What, How, Why Bother? Academic Exchange Quarterly, 15(2).

Lee, V. S. (2004) Teaching and learning through inquiry: A guidebook for institutions and instructors. Sterling: Stylus Publishing.

Lim, B. (2004) Challenges and Issues in Designing Inquiry on the Web. British Journal of Educational Technolog, Vol 35, No 5, 2004. pp. 627-643.

Liu, Yefeng; Alexandrova, Todorka; Nakajima, Tatsuo. (2011) Gamifying intelligent environments. Proceedings of the 2011 international ACM workshop on Ubiquitous meta user interfaces, Scottsdale, Arizona, USA.

Mott, B. W., Lester, J. C. (2006) Narrative-Centered Tutorial Planning for InquiryBased Learning Environments. Proceedings of the Eighth International Conference onIntelligent Tutoring Systems, vol. 4053, Berlin: Springer, pp. 675-684.

Nicholson, S. (2012) A User-Centered Theoretical Framework for Meaningful Gamification. Paper Presented at Games+Learning+Society 8.0, Madison, WI.

NMC. (2012) Perspectivas tecnológicas para o ensino fundamental e Médio Brasileiro de 2012 a 2017: Uma análise regional por NMC Horizon Project. Austin, Texas: The New Media Consortium Estados Unidos.

Prince, M., and R.M. Felder. (2006) Inductive teaching and learning methods: Definitions, comparisons, and research bases. Journal of Engineering Education 95 (2): 123-38.

Spronken-smith, R.A. (2007) Inquiry-based learning: Meaning, theoretical basis and use in tertiary education. Report prepared for the Ministry of Education, 32pp.

Vygotsky, L. S. Mind in Society: the Development of Higher Psychological Processes. Cambridge, MA.: Harvard University Press, 1978.

Yoder, M. (2005) Inquiry Based Learning Using the Internet: research, resources, WebQuests. In 19th Annual Conference on Distance Teaching and Learning.

Zichermann, Gabe. (2011) Gamification by Design. ISBN 1449397670. 150 pages. O'Reilly. 